\title{
New Approach to the Flexible Planning of Learning Process in High Education System
}

\author{
Badri Meparishvili ${ }^{1}$, Lela Turmanidze ${ }^{2}$ and Gulnara Janelidze ${ }^{1}$ \\ 1. Georgian Technical University, 77 Kostava st., 0175 Tbilisi, Georgia. \\ 2. Batumi Shota Rustaveli State University, 35 Ninoshvili st.,6010 Batumi, Georgia.
}

Received: January 03, 2015 / Accepted: February 01, 2015 / Published: May 25, 2015.

\begin{abstract}
Expediency of this work is conditioned by the inconsistency between the market requirement of the specialists and the planning process of high educational system. For solving this problem it is important to make consulting or expert system for flexible planning of teaching modules of every specialty. We make an attempt to consider this problem in two aspects: the prediction of market demand for planning taking into consideration of studies duration and scheduling of educational process. The prediction task consists in data acquisition of market requirement for each profession in discrete time interval to predict dynamic evolution of every specialty. The solution of the prediction task will be using to determination of prognostic quantity of students for each specialty. As regards the second aspect, it consists in finding a schedule of the teaching modules, i.e. the distribution of subjects in the semesters, keeping the total limits of credits, to update and adapt syllabus. In this paper, we present a genetic algorithm as a solution method for the modular scheduling problem. Genetic algorithms (GAs) allow a more general approach to the scheduling problem, which is rated using a fitness function. GA can be successfully applied to find optimized sequential schedules.
\end{abstract}

Keywords: Flexible Education, Genetic Algorithms, Scheduling.

\section{Introduction}

Problems of employment, long period education process, absence of coordination oriented on market demand makes necessary of working out Flexible High Education System. Each specialty with its disciplinary modules in it needs systematic structural and contextual modification. For solving this problem it is important to make consulting system. The system that can predict the dynamics of specialty evolution with the help of artificial intelligence methods. Recent changes in political geography in post-Soviet countries created a lot of problems. Georgia on its democratic way of socio-economic development has same problems, especially in the field of employment. Unfortunately, unemployment is a private problem in the regions, the institution of job-search solves the

Corresponding author: Lela Turmanidze, Batumi Shota Rustaveli State University, 35 Ninoshvili st.,6010 Batumi, Georgia. E-mail: turmanidzelela@gmail.com. problems only locally. Therefore we have huge number of unemployed with permanent job-searching problems, migration, demographic disbalances and risks of social and political stability. In spite of this higher education in Georgia is continuously growing, changing and developing.

\subsection{Formulation of the Problem}

The high education system (its long term duration) delays dynamics of market demands and increases not only welfare payment but also social funds for professional training of unemployed. In order to solve the above mentioned problems according to Bolognia declaration and Prague Communique the reforms in the system of high education should be based on the following:

(1) Standardize teaching programs and tests;

(2) General distance learning;

(3) Market oriented flexible, well-aimed high education. 
The priority of reform is working out market oriented, flexible high education system to meet requires of different universities and various specialties. Evaluation of specialties comprises the method of natural selection, i.e. the specialty that is not actual can not exit any more. If we consider branch model as a unity of several specialties, then the specialties should be considered as a unity of unity models that can be modified structurally and contextually. Taking into consideration present economic activity it is necessary to update modules and expand principle activities permanently.

\section{Experimental Section}

\subsection{Prediction Task}

Under the notion of flexible education is understood two independent problems: the prediction task and the planning and scheduling of educational process. In general, every statistical analysis and prediction is based on the statistics. The prediction task consists in data acquisition of market requirement for each profession $x_{i}\left(t_{k}\right)$ in discrete time interval (for example, month, quarter, year)

$$
t_{2}-t_{1}=t_{3}-t_{2}=\cdots=t_{N}-t_{N-1}=\Delta
$$

where: $i=1,2, \ldots, \mathrm{n}-$ is index of profession;

$\mathrm{k}=1,2, \ldots, \mathrm{N}$ - is index of planning time interval.

The tendency of variation of $x(t)$ can be described using the nonrandom function $f_{t r}(t)$, named the trend. Furthermore, there are also the others essential factors:

$\varphi(t)$ - the nonrandom function for description of periodical factors;

$\psi(t)$ - the nonrandom function for description of cyclical factors;

$\varepsilon(t)$ - the error or the random function for description of stochastic factors $\xi(1), \xi(1), \ldots, \xi(N)$.

As a rule, influence of above-mentioned factors on $x(t)$ is shown by the temporary series:

$$
\begin{aligned}
& x(t)=\chi(A) f_{t r}(t)+\chi(B) \varphi(t)+\chi(c) \psi(t)+\varepsilon(t) \\
& t=1,2, \ldots, N
\end{aligned}
$$

where: $G=A, B$ or $C$

$\chi(G)=\left\{\begin{array}{l}1, \text { if the factors } G \text { takes part in formation of } x(t) \\ 0, \text { in other case }\end{array}\right.$

The solution of the prediction task will be using to determination of prognostic value $x(n+\tau)$ for $\tau$ time, accordingly as the base for planning the number of students for each specialty, which e fitness-function as a quota of each specialty can be defined by:

$$
q_{i}=\frac{x_{i}}{\sum_{i=1}^{n} x_{i}}
$$

\subsection{Planning and Scheduling}

The particularity of this paper is the description of a new and efficient approach to solve the planning and scheduling problem for educational system especially flexible learning process using the Artificial Intelligence methods. In general planning and scheduling are common to many different domains. Although related and often tightly coupled, planning and scheduling are distinctly different activities. Planning is the construction of the project/process model and definition of constraints/objectives. Scheduling refers to the assignment of resources to activities (or activities to resources) at specific points in, or durations of, time. The definition of the problem is thus primarily a planning issue, whereas the execution of the plan is a scheduling issue. Yet planning and scheduling are coupled; the performance of the scheduling algorithm depends on the problem formulation, and the problem formulation may benefit from information obtained during scheduling.

In our case, it can be selected PERT, which is a method of characterizing precedence relationships between tasks and estimates of task requirements. In project scheduling problems, a single project consists of a set of tasks, or activities. The tasks have precedence relationships, i.e. some tasks cannot be started until their predecessors have been completed, and the tasks have resource requirements and the resources are limited. 


\subsection{Model}

As the high educational system is a particular branch, the planning and scheduling process will be a few different from above-mentioned standard conception. The module (of discipline) scheduling problem (MSP), may be described as follows: given $N$ $(i=1,2, \ldots, N)$ specialty, each composed of several $M$ $(j=1,2, \ldots, M)$ modules. The relations between them are of type "many to many". Every module can be characterize by: the priorities, the curriculum and programs, the constraints (inferior limit $C_{j}^{\text {min }}$ and upper limit $C_{j}^{\operatorname{mx}}$ ) of credit. Moreover, there is the interdisciplinary matrix (IDM), which describes interdependence between the modules i.e. module dependence graph (MDG). From one's part, the curriculum enclose the total limits of credit $L_{s}$ for each academic half-year.

MDG can be represented by a original directed graph $G(V, E)$. Each node $n_{i} \in V, i=1, \ldots, N$ corresponds to begin or end of the semesters. Each edge $e \in E$ corresponds to the modules. Figure 1 illustrates the general mode of graphical interpretation high educational process.

The problem consists in finding a schedule of the modules, i.e. the distribution of modules (taking into account theirs credits $C_{j}$ ) in the semesters keeping the total limits of credits. The conceptual model of the MSP can be described the following way:

$$
\begin{gathered}
C_{j}^{\min } \leq C_{j} \leq C_{j}^{\max } \\
\sum_{j=1}^{M} C_{j}=L_{s} \mid s \in S
\end{gathered}
$$

\subsection{Solution Method}

In this paper, we present a genetic algorithm as a solution method for the modular scheduling problem. Genetic algorithms are an effective method to solve complex optimization problems. Genetic algorithms allow a more general approach to the scheduling problem. They are based on evolution in the biological

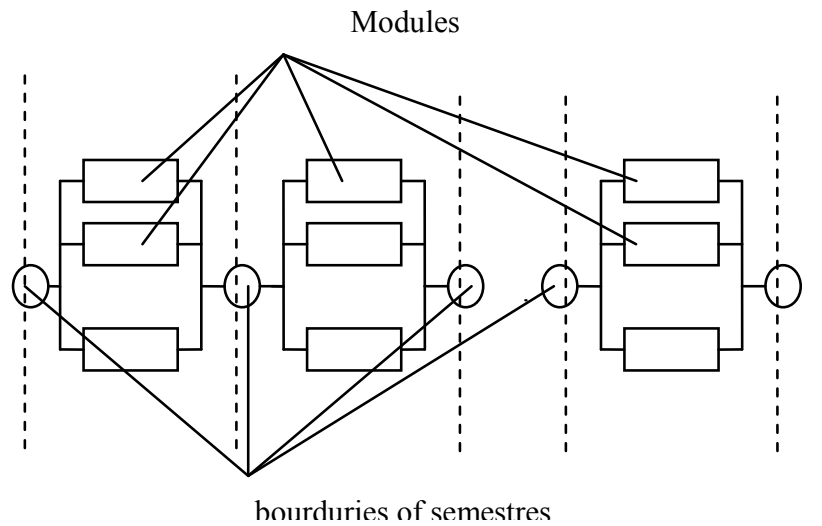

Fig. 1

sense and operate on a set of strings (chromosomes) the so-called population, where a string represents a possible solution for the parameters of the scheduling problem. New solutions are generated using genetic operators. The chromosomes are rated using a fitness function. The members of the next generation are chosen based on the fitness. GA have been successfully applied to find optimized sequential schedules.

Genetic algorithms are adaptive methods, which may be used to solve search and optimization problems [2,3]. To apply a genetic algorithm to a scheduling problem we must first represent it as a genome. One way to represent a scheduling genome in our population is to defines a parallel teaching modules in each semester [4].

To make sure that our genome is a feasible solution we must take care that it obeys our constraints. We generate an initial population using random schedules i.e. modules taking into consideration the priorities and the constraints of credit. Then we take this initial population and cross it combining genomes with a small amount of randomness. The offspring of this combination is selected based on a fitness function that includes our constraints.

We let this process continue until we find a solution for all academic year. The genetic algorithm is responsible for evolving the chromosomes which represent the priorities of the modules and credits. For each chromosome the following three phases are applied: 


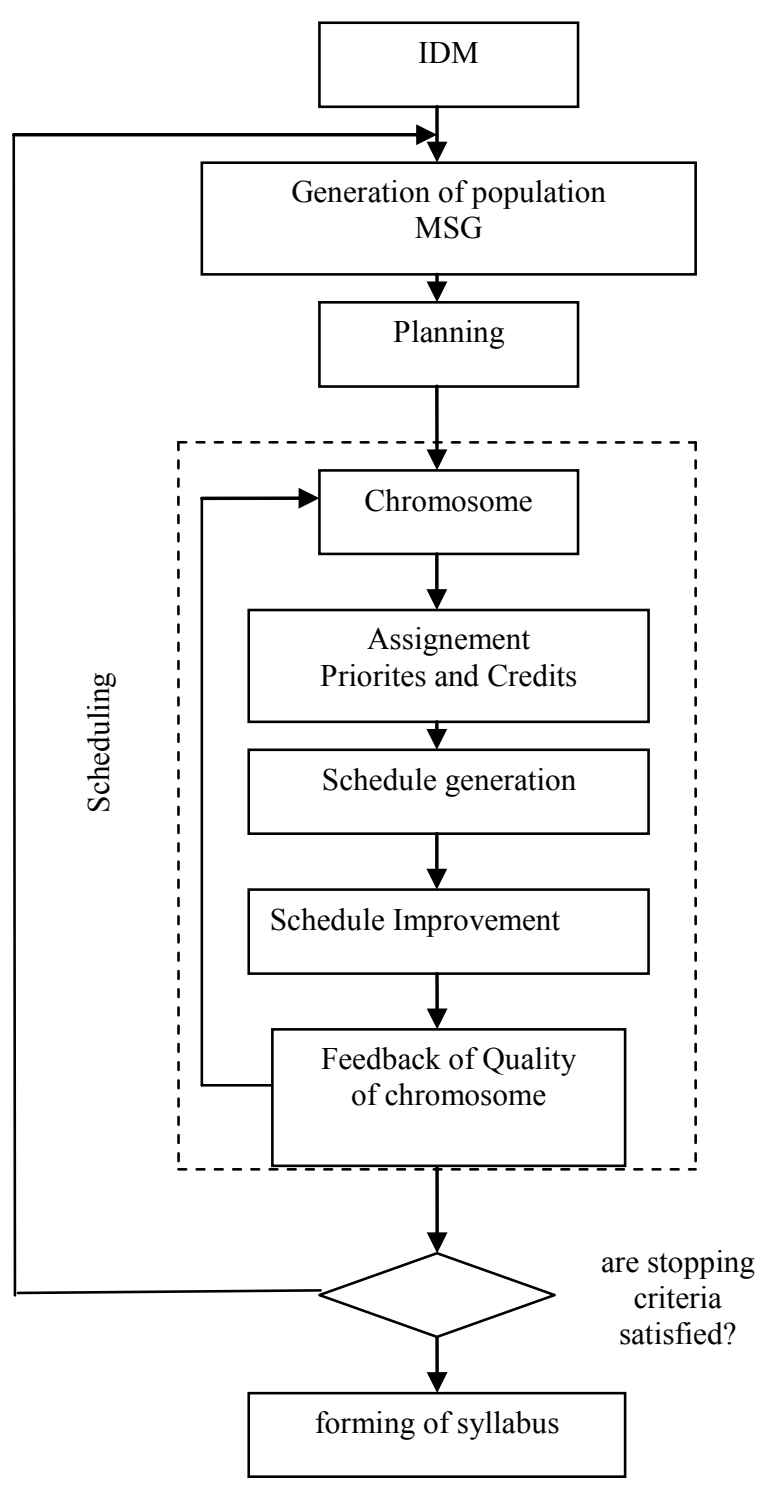

Fig. 2

- Representation of priorities and credits. This phase is responsible for transforming the chromosome supplied by the genetic algorithm into the priorities of the modules and credits.

- Schedule Generation. This phase makes use of the priorities and the credits defined in the first phase and constructs parameterized active schedules.

- Schedule Improvement. This phase makes use of a local search procedure to improve the solution obtained in the schedule generation phase.

After a schedule is obtained the corresponding quality is feedback to the genetic algorithm. Figure 2 illustrates the sequence of steps applied to each chromosome generated by the genetic algorithm [5].

\section{Results and Discussion}

Before a genetic algorithm can be run, a suitable representation for the problem must be devised. A fitness function is also required, which assigns a figure of merit to each encoded solution. During the run, parents must be selected for reproduction, and recombined to generate offspring. It is assumed that a potential solution to a problem may be represented as a set of parameters. These parameters (known as genes) are joined together to form a string of values (chromosome).

In genetic terminology, the set of parameters represented by a particular chromosome is referred to as an individual. The fitness of an individual depends on its chromosome and is evaluated by the fitness function. The individuals, during the reproductive phase, are selected from the population and recombined, producing offspring, which comprise the next generation. Parents are randomly selected from the population using a scheme, which favors fitter individuals. Having selected two parents, their chromosomes are recombined, typically using mechanisms of crossover and mutation. Mutation is usually applied to some individuals, to guarantee population diversity.

\section{Conclusions}

This paper presents a new approach to the planning of learning process in the different universities based on the artificial intelligence methods, particularly genetic algorithms. In the presentation the elaboration of consulting for flexible high education system is argumented in order to predict dynamic evolution of every specialty oriented on market demand. Its functional goal is to predict dynamic evolution of each specialty oriented on market demands, on one hand, and to update and adapt syllabus of teaching modules accordingly, on the other. 


\section{References}

[1] Aivazian S.A., Mkhitarian V.S. Applied statistics and fundamentals of econometric. M.: UNITI, (1998).

[2] Goldberg, D.E., (1989). Genetic Algorithms in Search Optimization and Machine Learning, Addison-Wesley.

[3] Beasley, D., Bull, D.R. and Martin, R.R. (1993). An Overview of Genetic Algorithms: Part 1, Fundamentals, University Computing, Vol. 15, No.2, pp. 58-69, Department of Computing Mathematics, University of
Cardiff, UK.

[4] Gonçalves, J.F. A Hybrid Genetic Algorithm for the Job Shop Scheduling Problem. AT\&T Labs Research Technical Report TD-5EAL6J, September 2002. pp. 113-137, (in Portuguese).

[5] Ediberidze, A. and Meparishvili, B. (2008). Several approaches to flexible high education system in Georgia. EUNIS 2008 International Conference "Visions for IT in Higher Education", Arhus, Denmark, 24-27 June, 2008. 114-115pp. 